\title{
IMAGES IN CLINICAL MEDICINE
}

Lindsey R. Baden, M.D., Editor

\section{Geographic Tongue}
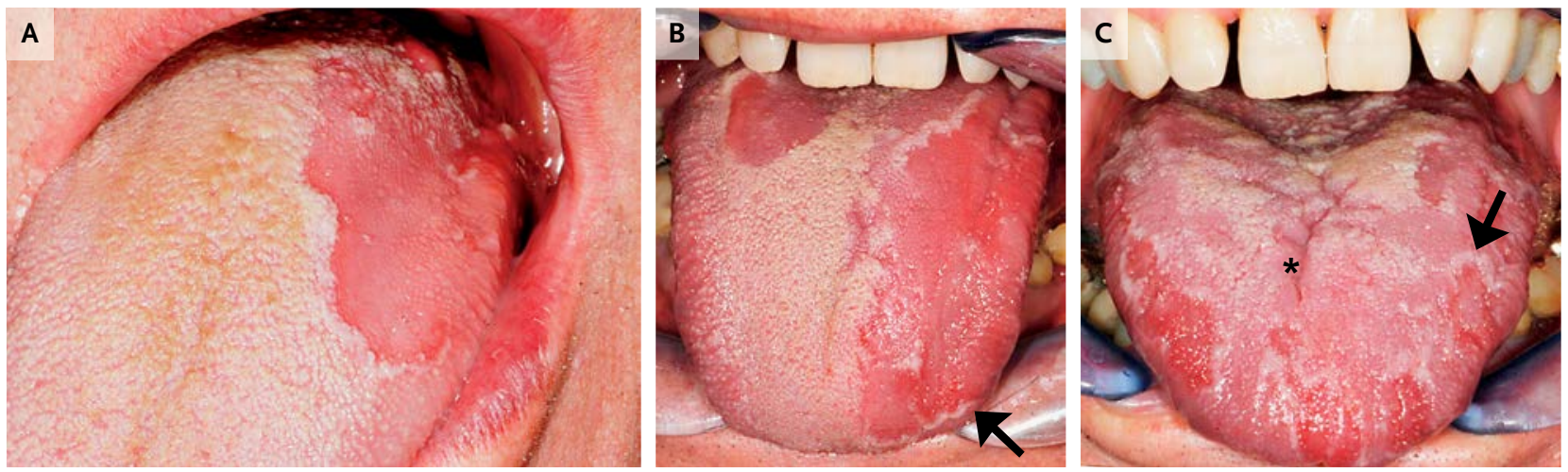

Elena Varoni, D.D.S., Ph.D.

Università degli Studi di Milano Milan, Italy

elena.varoni@unimi.it

Sem Decani, D.D.S.

Azienda Ospedaliera San Paolo Milan, Italy
73-YEAR-OLD MAN WAS REFERRED TO THIS CLINIC BY HIS PRIMARY CARE physician, who noted an unusual red lesion that had been moving across the dorsum of the man's tongue for approximately 3 months. The patient was a former smoker who was being treated for essential hypertension, hypercholesterolemia, and hyperuricemia. On oral examination, a well-defined, flat, rounded red area could be observed on the lingual dorsum; a mild coating of plaque could also be seen on the tongue (Panel A). A diagnosis of geographic tongue, also known as benign migratory glossitis, was made on the basis of the patient's clinical history and the pathognomonic sign of the migrant red lingual patch. Geographic tongue, which affects approximately $2 \%$ of the general population, is a benign condition associated with local inflammation and the absence of differentiation of the filiform papillae. Patients with geographic tongue typically present with migrant oval red areas, surrounded by a well-defined white rim. In our patient, the white rim was more clearly visible at follow-up visits at 8 months (Panel B, arrow) and 20 months (Panel C, arrow). Fissured tongue (Panel C, asterisk), which was modest in this patient, often accompanies this condition. Geographic tongue is usually asymptomatic, as it was in this case, but symptoms can include pain or a burning sensation that is triggered by eating spicy or acidic foods. When asymptomatic, geographic tongue does not require treatment. In our patient, geographic tongue was still present at a 32-month follow-up visit.

DOI: 10.1056/NEJMicm1502932

Copyright @ 2016 Massachusetts Medical Society. 